\title{
ACR-AAPM-SIIM Practice Guideline for Digital Radiography
}

\author{
Katherine P. Andriole • Thomas G. Ruckdeschel • \\ Michael J. Flynn • Nicholas J. Hangiandreou • A. Kyle Jones • \\ Elizabeth Krupinski • J. Anthony Seibert • S. Jeff Shepard • \\ Alisa Walz-Flannigan • Tariq A. Mian • Matthew S. Pollack
}

Published online: 20 September 2012

(C) Society of Imaging Informatics in Medicine and the American College of Radiology 2012

The American College of Radiology, with more than 30,000 members, is the principal organization of radiologists, radiation oncologists, and clinical medical physicists in the United States. The College is a nonprofit professional society whose primary purposes are to advance the science of radiology, improve radiologic services to the patient, study the socioeconomic aspects of the practice of radiology, and encourage continuing education for radiologists, radiation oncologists, medical physicists, and persons practicing in allied professional fields.

The American College of Radiology will periodically define new practice guidelines and technical standards for radiologic practice to help advance the science of radiology and to improve the quality of service to patients throughout the United States. Existing practice guidelines and technical standards will be reviewed for revision or renewal, as appropriate, on their fifth anniversary or sooner, if indicated.

Each practice guideline and technical standard, representing a policy statement by the College, has undergone a thorough consensus process in which it has been subjected to extensive review, requiring the approval of the Commission on Quality and Safety as well as the ACR Board of Chancellors, the ACR Council Steering Committee, and the ACR Council. The practice guidelines and technical standards recognize that the safe and effective use of diagnostic and therapeutic radiology requires specific training, skills, and techniques, as described in each document. Reproduction or modification of the published practice guideline and technical standard by those entities not providing these services is not authorized.

\section{K. P. Andriole}

Department of Radiology, Brigham and Women's Hospital, Harvard Medical School,

Boston, MA, USA

\section{T. G. Ruckdeschel}

Alliance Medical Physics LLC,

Alpharetta, GA, USA

\section{J. Flynn}

Department of Radiology, Henry Ford Health Systems,

Detroit, MI, USA

N. J. Hangiandreou $\cdot$ A. Walz-Flannigan Department of Radiology, Mayo Clinic, Rochester, MN, USA

\section{A. K. Jones}

Department of Imaging Physics, MD Anderson Cancer Center, Houston, TX, USA
E. Krupinski $(\square)$

Department of Medical Imaging, University of Arizona,

Tucson, AZ, USA

e-mail: Krupinski@radiology.arizona.edu

\section{J. A. Seibert}

Department of Imaging, University of California Davis health System,

Sacramento, CA, USA

\section{S. J. Shepard}

Department of Imaging Physics, UT MD Anderson Cancer Center, Houston, TX, USA

T. A. Mian

Medical Radiation Physics, Inc, Scottsdale, AZ, USA

M. S. Pollack

Warren Radiology Associates,

Phillipsburg, NJ, USA 


\section{Preamble}

These guidelines are an educational tool designed to assist practitioners in providing appropriate radiologic and radiation oncology care for patients. They are not inflexible rules or requirements of practice and are not intended, nor should they be used, to establish a legal standard of care. For these reasons and those set forth below, the American College of Radiology cautions against the use of these guidelines in litigation in which the clinical decisions of a practitioner are called into question.

The ultimate judgment regarding the propriety of any specific procedure or course of action must be made by the physician or medical physicist in light of all the circumstances presented. Thus, an approach that differs from the guidelines, standing alone, does not necessarily imply that the approach was below the standard of care. To the contrary, a conscientious practitioner may responsibly adopt a course of action different from that set forth in the guidelines when, in the reasonable judgment of the practitioner, such course of action is indicated by the condition of the patient, limitations of available resources, or advances in knowledge or technology subsequent to publication of the guidelines. However, a practitioner who employs an approach substantially different from these guidelines is advised to document in the patient record information sufficient to explain the approach taken.

The practice of medicine involves not only the science but also the art of dealing with the prevention, diagnosis, alleviation, and treatment of disease. The variety and complexity of human conditions make it impossible to always reach the most appropriate diagnosis or to predict with certainty a particular response to treatment.

Therefore, it should be recognized that adherence to these guidelines will not assure an accurate diagnosis or a successful outcome. All that should be expected is that the practitioner will follow a reasonable course of action based on current knowledge, available resources, and the needs of the patient to deliver effective and safe medical care. The sole purpose of these guidelines is to assist practitioners in achieving this objective.

\section{Keypoints}

The intent of this document is to provide guidance and assistance in the understanding and clinical use of digital radiography equipment in order to deliver optimal image quality at an appropriate minimal radiation dose and to ultimately provide excellent safety and care for patients undergoing digital radiography examinations. An introduction to the realm of digital radiography, including definitions, is presented in "Introduction and Definition." As new capabilities and complexities arise with digital imaging devices, the qualifications and responsibilities of personnel -including the physician, medical physicist, radiologist assistant, radiologic technologist, and image management specialist - are affected as outlined in section II.

\section{Introduction and Definition}

This guideline was developed collaboratively by the American College of Radiology (ACR), the American Association of Physicists in Medicine (AAPM), and the Society for Imaging Informatics in Medicine (SIIM).

Increasingly, medical imaging and patient information are being managed using digital data during acquisition, transmission, storage, display, interpretation, and consultation. The management of these data during each of these operations may have an impact on the quality of patient care.

"CR" and "DR" are the commonly used terms for digital radiography detectors. CR is the acronym for computed radiography, and DR is an acronym for digital radiography. CR uses a photostimulable storage phosphor that stores the latent image, which is subsequently read out using a stimulating laser beam. It can be easily adapted to a cassette-based system analogous to that used in screen-film (SF) radiography. Historically, the acronym DR has been used to describe a flat-panel digital X-ray imaging system that reads the transmitted X-ray signal immediately after exposure with the detector in place. Generically, the term CR is applied to passive detector systems, while the term DR is applied to active detectors.

This guideline is applicable to the practice of digital radiography. It defines motivations, qualifications of personnel, equipment guidelines, data manipulation and management, and quality control (QC) and quality improvement procedures for the use of digital radiography that should result in high-quality radiological patient care.

In all cases for which an ACR practice guideline or technical standard exists for the modality being used or the specific examination being performed, that guideline or standard will continue to apply when digital image data management systems are used.

\section{Qualifications and Responsibilities of Personnel}

Individuals performing digital radiography procedures must be appropriately trained in the proper use of the imaging equipment and must have the appropriate level of knowledge necessary to obtain optimal information for each requested procedure. In all cases, the operator should be a physician, a radiologist assistant, a licensed and/or registered radiologic technologist, or a radiation therapist. The radiologist assistant, 
technologist, or radiation therapist must be operating under the direct supervision of a qualified licensed physician.

\section{Physician}

1. Physicians using digital radiography should understand the basic technology of image acquisition, transmission, manipulation, processing, archiving, retrieval, and display, including the strengths, weaknesses, and limitations of different methods. They should be knowledgeable in how to optimally utilize the image viewing equipment. Where appropriate, the interpreting physician must be familiar with the principles of radiation protection, the hazards of radiation exposure to both patients and radiological personnel, and patient and personnel monitoring requirements. The physician performing the official interpretation must be responsible for the quality of the images being reviewed and understand the elements of QC of digital image management systems. ${ }^{1}$

2. The physician must demonstrate qualifications as delineated in the appropriate ACR Practice Guideline or Technical Standard for the particular diagnostic modality being interpreted.

3. The physician should have a working knowledge of those portions of the digital imaging chain from acquisition to display that affect image quality and that have the potential for producing artifacts.

\section{Qualified Medical Physicist}

A qualified medical physicist must be on site or available as a consultant.

A qualified medical physicist is an individual who is competent to practice independently in one or more of the subfields in medical physics. The American College of Radiology considers certification, continuing education, and experience in the appropriate subfield(s) to demonstrate that an individual is competent to practice one or more of the subfields in medical physics and to be a qualified medical physicist. The ACR strongly recommends that the individual be certified in the appropriate subfield(s) by the American Board of Radiology, the Canadian College of Physics in Medicine, or by the American Board of Medical Physics.

The appropriate subfield of medical physics for this standard is Diagnostic Medical Physics. (Previous medical

\footnotetext{
${ }^{1}$ The ACR Rules of Ethics state: "It is proper for a diagnostic radiologist to provide a consultative opinion on radiographs and other images regardless of their origin. A diagnostic radiologist should regularly interpret radiographs and other images only when the radiologist reasonably participates in the quality of medical imaging, utilization review, and matters of policy which affect the quality of patient care."
}

physics certification categories including radiological physics, diagnostic radiological physics, and diagnostic imaging physics are also acceptable.)

A qualified medical physicist should meet the ACR Practice Guideline for Continuing Medical Education (ACR Resolution 17, adopted in 1996-revised 2012, Resolution 42).

\section{Registered Radiologist Assistant}

A registered radiologist assistant is an advanced level radiographer who is certified and registered as a radiologist assistant by the American Registry of Radiologic Technologists after having successfully completed an advanced academic program encompassing an ACR/American Society of Radiologic Technologists (ASRT) radiologist assistant curriculum and a radiologist-directed clinical preceptorship. Under radiologist supervision, the radiologist assistant may perform patient assessment, patient management and selected examinations as delineated in the Joint Policy Statement of the ACR and the ASRT titled "Radiologist Assistant: Roles and Responsibilities" and as allowed by state law. The radiologist assistant transmits to the supervising radiologists those observations that have a bearing on diagnosis. Performance of diagnostic interpretations remains outside the scope of practice of the radiologist assistant (ACR Resolution 34, adopted in 2006).

Radiologic Technologist and Radiation Oncology Therapist

The radiologic technologist or radiation oncology therapist must be certified by the appropriate registry and/or possess unrestricted state licensure.

1. The individual must meet the qualification requirements of any existing ACR practice guideline or technical standard for acquisition of a particular examination.

2. He or she must be trained to properly operate those portions of the image data management system with which he or she must routinely interact. This training should include as appropriate:
(a) Image acquisition technology.
(b) Image processing protocols.
(c) Proper selection of examination specific options.
(d) Image evaluation.
(e) Radiation dose indicators.
(f) Patient safety procedures.

\section{Imaging Informatics Professional}

The imaging informatics professional should be qualified to assess and provide problem-solving input, initiate repair, and coordinate system-wide maintenance programs to assure sustainable high image quality and system function. 
The responsibilities and experience for an imaging informatics professional include:

1. Maintenance of the network for all informatics systems, e.g., radiology information system, picture archiving and communication system (PACS), speech recognition systems, and computer servers and desktops.

2. Maintenance of the integrity of system databases to ensure continuous and accurate operation of the informatics systems.

3. Coordination of the interaction and functionality of all data entry and management systems with the necessary radiology applications, programs, and databases.

4. Knowledge of computer systems using all possible operating systems (Windows, Unix/Linux, Mac), data communications standards and equipment, network protocols, database management, internet protocols, and systems analysis methods and design.

A qualified imaging informatics professional is an individual who is competent to practice independently in the areas of informatics listed above. He or she should have a minimum of a bachelor's degree in computer science or equivalent. Continuing education and experience in imaging informatics demonstrate that an individual is competent to practice as an imaging informatics professional. Certification through the American Board of Imaging Informatics (ABII) can be used to validate an individual's qualification as a qualified imaging informatics professional.

\section{Equipment Specifications}

Specifications for equipment used in digital image data management will vary depending on the application and the individual facility's needs but in all cases should provide image quality and availability appropriate to the clinical needs.

\section{Image Availability and Information Standardization}

To ensure the enterprise-wide availability of features and performance when purchasing digital radiographic and connected equipment, consideration of the manufacturers' statements of conformance with the current ACR-National Electrical Manufacturers Association Digital Imaging and Communications in Medicine (DICOM) standard is strongly recommended. Also consideration of periodic upgrades incorporating the expanding features of that standard should be part of the ongoing QC program. Compliance with the Radiological Society of North America and Healthcare Information and the Management Systems Society Integrating the Healthcare Enterprise Initiative, as embodied in the available technical frameworks, is also strongly recommended for all new equipment acquisitions.
Specifications and usage guidelines related to standards and interoperability include:

1. Digital radiographic devices must provide images that conform to the DICOM standard "CR" or "DX" service class objects. These objects' header fields specify information such as accession number, patient name, identification number, date and time of examination, name of facility or institution of acquisition, type of examination, patient or body part orientation (e.g., right, left, superior, and inferior), amount and method of data compression, and total number of images acquired in the study.

2. The use of DICOM modality work lists is recommended to help ensure the quality and accuracy of the information captured in the DICOM header.

3. The use of the DICOM "DX" service class object is recommended instead of the more limited "CR" object for digital radiography [1].

4. It is recommended to use DICOM grayscale softcopy presentation state (GSPS) objects to transmit annotations, shutter, and display lookup tables (LUTs) [2]. Where GSPS is not available or not supported by a PACS, the use of a volume-of-interest lookup table (VOI-LUT) within the "CR" or "DX" service class object is suggested.

5. Details related to image acquisition such as tube potential (in peak kilovoltage) and tube current (in milliamperes) exposure time, beam filtration, and radiation exposure indicators should be recorded in the DICOM header, particularly for cassette-based and cassette-less DRs that have a direct interface to the $\mathrm{X}$-ray generator.

Acquisition

Image acquisition should be performed in accordance with the ACR-SPR Practice Guideline for General Radiography or examination-specific ACR Practice Guidelines.

\section{Use of Antiscatter Grids}

Scattered radiation diminishes contrast in radiography, reducing the available dynamic range of X-ray intensities at the beam exit side of the patient. It also decreases the SNR because it contains no signal but does contain X-ray quantum noise.

(a) Scanned slot DR detectors possess inherent scatter rejection capability and do not require the use of a grid.

(b) Area detectors employed in digital radiography should be used with an antiscatter grid in clinical imaging situations where scatter dominates, and usually at patient thicknesses in excess of $10 \mathrm{~cm}$. Grids are not 
required when imaging small pediatric patients or small body parts such as extremities. Grid uses for specific radiographic examinations are discussed in examination-specific ACR Practice Guidelines and Technical Standards.

(c) When using stationary grids, consider the use of a high strip frequency grid of $>60$ lines/cm to avoid grid aliasing patterns caused by insufficient sampling. Consultation with a qualified medical physicist or the vendor regarding an appropriate grid is recommended.

(d) When using antiscatter grids with digital detectors, an increase in exposure (in milliamperes per second) is necessary only to compensate for attenuation of primary radiation by the grid.

\section{Exposure Indicators for Digital Radiography}

With the wide latitude of digital radiographic devices, the visual appearance of brightness and contrast in radiographic images is not a good indicator of the appropriateness of the exposure delivered to the image receptor. Digital radiographic devices can create satisfactory images over a wide range of input exposures, including exposures that are significantly greater than are clinically necessary. For images acquired with higher than desired detector exposure, the patient may receive excessive radiation dose. Images acquired at lower than required exposures may have unacceptably high noise levels. For these reasons, digital radiography manufacturers should provide a number (i.e., an exposure indicator, or air kerma indicator) that reflects the estimated exposure reaching the detector. This can provide feedback to technologists on the appropriateness of a chosen imaging technique, or to a practice in determining its standard techniques.

Currently, most digital radiography manufacturers provide an exposure indicator that is displayed alongside the image on the acquisition workstation, providing feedback to the technologist about the acquisition technique. This information should also be transmitted to the PACS in the DICOM header of the image. Generally, display of the acquisition technical factors has been of greatest use to the radiologic technologist and of less utility to the interpreting physician. However, should it be desired, it should be configurable for the exposure indicator to be shown along with the image on either the technologist's QC display or the radiologist's diagnostic display.

Today, the various numerical exposure indicators used by the different digital radiography manufacturers are inconsistent and not easily comparable. The AAPM formed Task Group 116 to address the lack of a uniform exposure indicator on digital radiography images [3]. Its report, "Recommended Exposure Indicator for Digital Radiography," defines standardized radiation exposure conditions from which a relative exposure indictor can be defined.
The International Electrotechnical Commission (IEC) released an international standard for the exposure index of digital X-ray imaging systems [4] that is consistent with AAPM Report 116 [3]. Presently, new DR systems and upgraded software versions for existing equipment are incorporating the IEC standard. In addition to the traditional exposure index, a deviation index is reported that describes how the exposure index deviates from a target value. Users should review the target values for all views of all body parts that the system will be used to image. Target values should be selected to minimize the exposure to the patient while providing diagnostic images (i.e., with sufficiently low noise) for interpretation.

\section{Exposure Creep}

In digital radiography, excessive exposure to the detector can produce high-quality images with improved noise properties. Unless there is an understanding that these higherquality images comes at the cost of increased patient exposure, and strategies are in place to control patient exposure, a radiologic practice may experience "exposure creep" [5]. Exposure creep results when there is negative radiologist feedback for high-noise images made with low exposures, but a lack of negative feedback or even positive feedback for low-noise images made with high exposures. As technologists respond to this feedback, patient exposures may gradually increase over time. The qualified medical physicist should monitor for exposure creep on a consistent, ongoing basis, as it may be a recurring phenomenon.

A method to prevent exposure creep is to develop validated radiographic technique charts as a function of patient size for all performed examinations. If these factors are entered into the anatomical programming of state-of-the-art $\mathrm{X}$-ray generator controls, the technologist can simply select the radiological examination and patient size at the beginning of an examination. If the technologists uses these techniques, the facility is assured of the use of standard radiographic technique factors and standard radiation exposure whether the examination is performed with automatic exposure control (AEC) or manual techniques [6].

\section{Radiographic Technique Considerations for Digital Radiography}

(a) Determining Proper Exposure Factors for Standard Examinations

Exposure (technique) charts are part of the standard of care expected by the Joint Commission and are required by law in many states. It is necessary to check state and/or local regulations for any specific requirements. Computation of estimates for entrance skin exposures for these charts may also be required. 
Because of the wide latitude of digital image receptors and the availability of image processing to alter the brightness and contrast of images, the visual appearance of images can be made similar over a wide range of acquisition techniques. The primary effects of modifying an acquisition technique are changes in:

1. The appearance of noise in the image.

2. The exposure duration and potential for patient motion artifacts.

3. Patient radiation exposure.

4. Potential artifacts (in DR) related to detector saturation and image lag.

Exposure technique charts must be tailored for each digital radiography $\mathrm{X}$-ray system and detector combination [7]. There is considerable variability in image receptor response owing to varying scatter sensitivity, the use of grids with different grid ratios, collimation, beam filtration, the choice of kilovoltage, source-to-image distance, and image receptor size.

Exposure technique charts are typically provided by equipment manufacturers, either as prescriptions for manual radiographic technique or programmed examination specific options in the X-ray generator. Many digital radiography systems also permit the choice of several speed (exposure) classes. Technique factors for each clinically used speed class should be constructed. In addition, exposure charts should be designed to function over a wide range of adult patient sizes [8] and over the even wider range of pediatric patient sizes.

Techniques can be modified to suit particular clinical needs or patient subgroups, and this process is best done in collaboration between a qualified medical physicist and radiologists. The aim of modifying a radiographic technique for a particular clinical purpose would be to:

1. Decrease the appearance of noise in an image to better suit the intended diagnostic task.

2. Reduce patient exposure for images where higher noise levels are acceptable.

3. Reduce patient motion artifacts.

(b) Automatic Exposure Control

In CR, AEC is frequently used with wall and undertable Bucky devices in emergency, inpatient, and outpatient radiology department settings. AEC units are designed to turn off the X-ray generator when an appropriate exposure level has been received at the image receptor. They work well when the device is properly calibrated and the body part being imaged is positioned properly over the active $\mathrm{AEC}$ region. AEC may fail if positioning is poor or if material contained within the sensitive area of the AEC device is of a different density than tissue, for example a prosthesis in the hip of a patient.

AEC devices are energy dependent and may require calibration at multiple tube potential values to function properly over a wide range of patient sizes. Some X-ray generators have preprogrammed energy response curves and may not work as well with CR as with conventional SF receptors if they were manufactured to be matched to typical SF energy and scatter characteristics. In this situation, the AEC device should be recalibrated for use with CR $[9,10]$.

In DR, the AEC device is typically built into the image receptor assembly, and it works in a manner similar to the systems used in SF radiography. Proper calibration should still be verified by a qualified medical physicist. Also, the selection of the appropriate sensitivity setting by examination should be verified.

(c) Image Repeat for Improper Exposure

As described in "Exposure Indicators for Digital Radiography," vendors should provide exposure indicators with images. These can be used to provide an expected range of exposure indices for a given examination type. These ranges can be used to develop exposure technique charts or to monitor for exposure creep in a practice. However, an exposure indicator that is higher or lower than what is typical or expected should not be cause for immediately repeating an image without careful review of the image.

It is important for the radiologist and technologist to review improperly exposed radiographs to develop an understanding for which radiographs should be repeated. Technologists may need to be provided with the appropriate image viewing resources (see "Display Capabilities") and training to determine if a radiograph needs to be repeated on the basis of image noise or receptor saturation. For the different classes of rejected images, it is useful to provide guidance to the technologist on how to correct an improperly exposed radiograph, either through processing or by modifying exposure technique.

(d) Pediatric Imaging Considerations

As with all imaging examinations involving ionizing radiation, digital radiography should be performed using the lowest possible radiation exposure to the patient that is consistent with image quality requirements. This is especially important when imaging pediatric patients who are believed to be up to ten times more sensitive to ionizing radiation than adults [11, 12]. However, reducing exposure without assessing image quality increases the possibility of unacceptably 
high quantum noise. Also, the appropriate reduction in exposure to the image receptor depends on how well the digital system is optimized for pediatric imaging and on the nature of the particular condition being diagnosed [8].

Commitment and effort on the part of staff are required to achieve exposure reduction in pediatric imaging. First, the multiple X-ray machines of a given type in a department require validation, calibration, and matching to ensure the same exposure for similar radiographic technique parameters. The actual exposure required to produce clinically acceptable images for a given digital receptor is a function of the digital receptor's detective quantum efficiency. The goal is to produce similar image quality in all images regardless of the device chosen and regardless of the size of the pediatric patient. Second, all CR readers and/or DR image receptors should be adjusted to provide a uniform response to the recorded X-ray image pattern in space. Third, all monitors on all diagnostic display workstations should be tested and calibrated to provide a uniform appearance of displayed images. These topics are addressed in more detail elsewhere [10].

Pediatric patients present unique challenges when imaged with digital radiography. Patients range in size from the neonate to the young adult, requiring a wide range of radiographic technique factors and a digital image receptor that properly processes an X-ray pattern in space that may or may not cover the entire image receptor. The decision to use an antiscatter grid must be carefully considered as a function of patient size.

Standard positioning aids used to immobilize pediatric patients may generate unacceptable artifacts when used with digital image receptors. Because the X-ray pattern resulting from a pediatric chest has a different dynamic range and other characteristics [12], compared with an adult chest, image processing and display parameters used by the digital acquisition device to properly display the digital image may need to be configured as a function of patient size. Image processing and display parameters may require further alteration if gonadal shielding or orthopedic implants appear in the displayed image. Finally, because scoliosis examinations are common in children, the digital image receptor must provide an efficient method to generate images up to 36 in. in length without doubly exposing some sections of the patient's anatomy. Additional information regarding pediatric imaging protocols can be found at the Image Gently website: www.imagegently.org.

\section{Image Processing}

For digital imaging, image processing (which is often proprietary to the device manufacturer and not under the control of the radiologist) is used to determine display contrast by establishing the relationship between raw pixel values and grayscale levels. This results in the final "For Presentation" image for interpretation on either hardcopy film or softcopy displays.

\section{Preprocessing}

The image receptor on most digital radiography systems stores an electronic charge that is monotonically related to the amount of radiation energy absorbed. At this stage, the signal (charge) is a linear function of the incident radiation exposure. Preamplifiers and an analog-to-digital converter transform the charge from each detector element to an integer representing the raw data image value. Several corrections are applied to the raw image values to obtain values suitable for image processing. These corrections include interpolating bad pixels and to adjusting for nonuniformity.

DR system users should ask the supplier for information indicating the number and type of bad pixels that are being corrected. QC programs should include a process to report any new bad pixels that may develop during the lifetime of the system. Nonuniformity corrections generally require running a system utility program periodically during which uniform exposures are acquired. Users should identify the required procedure for each type of system being used and ensure that the procedures are being followed.

2. For-Processing Images

Most DR systems transform the preprocessed value to a value proportional to the logarithm of the input exposure. Logarithmic signals have the property that a fractional change in signal, due to the contrast of adjacent structures, produces a fixed change in the raw image value independent of subject penetration and input exposure. These values are the For-Processing images values and may be stored in DICOM image objects. The AAPM further recommends specific units for normalized ForProcessing image values [3].

In some operations, users may archive For-Processing images so that they may be processed at a later date. For example, when reading a current digital mammography image it may be desirable for a prior mammogram to be processed using the same method. Another use case involves CAD systems that require For-Processing images.

3. DR Image Processing Operations

Image processing operations are performed on ForProcessing images to obtain For-Presentation images with values suitable for display on a workstation monitor. 
All suppliers of DR equipment provide image processing software that can restore the sharpness of edges, enhance detail contrast for images with a wide range of input exposures, and reduce noise [13]. When properly implemented, image processing can restore the sharpness of edges without introducing artifacts. Detail contrast can be enhanced by multifrequency processing that equalizes image brightness over broad areas by operating on low spatial frequencies. Most recently, methods have been introduced to reduce high frequency image noise without adversely effecting image resolution. In general, the image processing from different systems can be adjusted to give similar presentation appearance for images of the same body part, as the desired appearance may be different in different facilities. The user should anticipate working with the applications specialists of their equipment manufacturer to configure the desired image processing looks.

The parameters used to process images need to be specifically determined for all body parts and views that will be encountered. In the past, this required significant effort by the user to establish the desired appearance for all views. Currently, most systems make these adjustments internally, with the user determining the generic characteristics of the presented image. For example, users may differ with respect to the amount of detail contrast enhancement that is desired.

4. DR Image Grayscale

As a part of the operations that transform ForProcessing images to For-Presentation images, the image values in the anatomical regions of interest, referred to as the values of interest (VOI), are identified and used to compute a LUT used to display the For Presentation values. Earlier systems applied the VOI-LUT in the DR systems and sent these image values within the DICOM object. As described in "Image Availability and Information Standardization" above, it is preferable that the VOI-LUT be sent and that the image display software transform the values at the workstation. This allows the user at the workstation to make further adjustment in the grayscale of the image. The VOI are also used to calculate the exposure index and associated deviation index that are used as indicators of proper radiographic technique (see "Exposure Indicators for Digital Radiography"). The edges of the collimated regions of the image should be recognized by the system and the regions outside of the collimators masked to prevent presentation of large bright regions to the radiologist, and to ensure accurate computation of the exposure indicator. It is preferable that this mask be encoded in the DICOM image as an overlay so that if needed it can be removed to see information that might be near the collimated edge, such as a marker.
Image Data Integrity and Transmission

1. Compression

Data compression may be performed to facilitate image transmission and storage. The type of medical image, the modality, and the objective of the study will determine the degree of acceptable compression. For more information on image compression, see the ACR-AAPM-SIIM Technical Standard for Electronic Practice of Medical Imaging.

2. Data Transmission

The environment in which imaging studies are to be transmitted will determine the types and specifications of the transmission devices used. In all cases, for official interpretation, the digital data received at the end of any transmission must have minimal loss of clinically significant information. The transmission system must also have adequate error-checking capability.

Additional consideration should be given for devices that rely on wireless networking capabilities. Such systems are increasingly common in digital radiography, especially those sued for bedside radiography. The wireless transmission of information may be between the digital detector and the acquisition workstation (i.e., wireless DR detectors) or between the acquisition workstation and the institutional PACS (i.e., bedside imaging) or both. Provisions should be made for:

(a) The prevention of signal interference and corruption between different systems communicating on the same wireless network.

(b) Appropriate signal encryption to protect PHI.

(c) Strategy for loss-prevention and image recovery for images which have interrupted transmission or corrupted transmission, especially for the communication between the detector and acquisition workstation which is less likely use a DICOM standard transaction with storage-commitment functionality.

For further details refer to the ACR-SIIM Practice Guideline for Electronic Medical Information Privacy and Security, and the ACR-AAPM-SIIM Technical Standard for Electronic Practice of Medical Imaging.

\section{Security}

See the ACR-SIIM Practice Guideline for Electronic Medical Information Privacy and Security.

4. Archiving and Retrieval, Reliability and Redundancy, and Work and Room Environmental and Ergonomic Considerations

See the ACR-AAPM-SIIM Technical Standard for Electronic Practice of Medical Imaging.

5. Display Capabilities

The consistent presentation of images across different workstations is essential for a quality imaging practice. 
Images are viewed by technologists during acquisition, by radiologists during interpretation, and by referring physicians as a part of patient care, and should have a similar appearance for all viewers. The following uses emphasize the importance of choosing appropriate display equipment and settings, as well as the importance of regular calibration and maintenance as part of a QC program.

(a) Image modifications are most often made by the technologist at the acquisition workstation. Technologists may modify image processing to try to match the appearance desired by radiologists. If the acquisition display does not meet the specifications for consistent presentation of images (as described in the ACR-AAPM-SIIM Technical Standard for Electronic Practice of Medical Imaging), or is not calibrated or maintained properly, this image modification process is particularly challenging.

(b) A primary method for a radiology technologist to discern whether a low-exposure image acquisition needs to be repeated is by comparison of the noise levels in the image with the desired diagnostic appearance. Visualizing image noise may be difficult on poor performing displays.

(c) Owing to the nature of use of point-of-care digital radiographic devices (i.e., DR mobile X-ray systems), they are often used for primary diagnostic tasks. Digital radiography manufacturers should provide highquality display options as outlined in the ACRAAPM-SIIM Technical Standard for Electronic Practice of Medical Imaging for displays on which diagnostic or primary interpretation tasks might be performed or one which images are modified.

Guidelines for viewing digital radiography images viewing can be found in the ACR-AAPM-SIIM Technical Standard for Electronic Practice of Medical Imaging and for mammography in the ACR-AAPM-SIIM Practice Guideline for Determinants of Image Quality in Digital Mammography.

\section{Documentation}

Physicians officially interpreting examinations ${ }^{2}$ using digital image data management systems should render reports in accordance with the ACR Practice Guideline for Communication of Diagnostic Imaging Findings.

\footnotetext{
$\overline{2}$ The ACR Medical Legal Committee defines official interpretation as that written report (and any supplements or amendments thereto) that attach to the patient's permanent record. In health care facilities with a privilege delineation system, such a written report is prepared only by a qualified physician who has been granted specific delineated clinical privileges for that purpose by the facility's governing body upon the recommendation of the medical staff.
}

If reports are incorporated into the data management system, they should be retrievable with the same timeliness and security as the imaging data.

\section{Radiation Safety in Imaging}

Radiologists, medical physicists, radiologic technologists, and all supervising physicians have a responsibility to minimize radiation dose to individual patients, to staff, and to society as a whole, while maintaining the necessary diagnostic image quality. This concept is known as "as low as reasonably achievable (ALARA)."

Facilities, in consultation with the medical physicist, should have in place and should adhere to policies and procedures, in accordance with ALARA, to vary examination protocols to take into account patient body habitus, such as height and/or weight, body mass index or lateral width. The dose reduction devices that are available on imaging equipment should be active; if not; manual techniques should be used to moderate the exposure while maintaining the necessary diagnostic image quality. Periodically, radiation exposures should be measured and patient radiation doses estimated by a medical physicist in accordance with the appropriate ACR Technical Standard (ACR Resolution 17, adopted in 2006 - revised in 2009, Resolution 11).

\section{Quality Control and Improvement, Safety, Infection Control, and Patient Education}

Policies and procedures related to QC and improvement, patient education, infection control, and safety should be developed and implemented in accordance with the ACR Policy on Quality Control and Improvement, Safety, Infection Control, and Patient Education appearing under the heading Position Statement on QC \& Improvement, Safety, Infection Control, and Patient Education on the ACR web site (http://www.acr.org/guidelines).

Any facility using a digital image data management system must have documented policies and procedures for monitoring and evaluating the effective management, safety, and proper performance of acquisition, digitization, processing, compression, transmission, display, archiving, and retrieval functions of the system. The QC program should be designed to maximize the quality and accessibility of diagnostic information (see Appendix).

\section{Quality Control Program}

A continuous QC program must be established for all digital radiography under the guidance of a qualified medical physicist. The qualified medical physicist, in 
consultation and accordance with the equipment manufacturer, applicable industry guidelines, and published standards, and with state and federal regulations, should determine the methodology and frequency of each test to be performed. An on-site radiologic technologist must be identified to be responsible for conducting routine QC.

1. Performance testing and monitoring of CR and DR equipment should include the recommendations for QC testing methods and frequencies contained in report of the AAPM Task Group No. 10: Acceptance Testing and Quality Control of Photostimulable Storage Phosphor Imaging Systems [14].

2. Guidance on performance testing and monitoring of official or primary interpretation display devices is available in the ACR-AAPM-SIIM Technical Standard for Electronic Practice of Medical Imaging.

\section{Key Aspects of Ongoing Quality Control}

The key aspects of ongoing QC include, but are not limited to:

1. Exposure analysis - data relating to patient exposures, including DAP or exposure indicators, should be collected and analyzed on a regular basis. Such data can be compared with published reference levels to identify areas for improvement. SF imaging is largely self-policing in terms of receptor exposure, and under and overexposed films are included in the rejected image analysis process.

2. Rejected image analysis - rejected image analysis is commonly performed in SF imaging departments but has been abandoned in many digital imaging departments, likely owing to the fact that physical images are not collected and digital images are easily deleted. While the number of images rejected owing to under and overexposure has been reduced in digital imaging, rejected image analysis is still critical, as positioning errors continue to be the most common reason for repeating images. Rejected image data should be collected and analyzed on a regular basis and corrective action taken when necessary.

3. Artifact identification - artifacts are not eliminated when changing from SF to digital imaging, and the types of artifacts that occur in digital radiography are different than those seen in SF imaging. Therefore, it is important that radiologic technologists and radiologists be taught to identify common digital imaging artifacts. In addition, radiologic technologists should carefully analyze each digital image for artifacts so that corrective action, including restriction of equipment use, can be taken to eliminate the artifact. For this reason, it is critical that the monitors used by the radiologic technologist to review images be suitable for this task.
The results of the QC program must be monitored annually by the qualified medical physicist. If measured values of QC parameters fall outside the control limits, the qualified medical physicist must initiate appropriate investigative or corrective actions. A qualified medical physicist should be available to assist in prescribing corrective actions for unresolved problems.

\section{Acceptance Testing}

Initial performance testing of imaging equipment must be performed by a qualified medical physicist upon installation and should be completed before first clinical use. This testing may be more comprehensive than periodic performance and compliance testing and should be consistent with current acceptance testing practices for digital radiography. Specific tests to be performed depend on the type of equipment and the manufacturer's recommendations. The AAPM has a document (AAPM Task Group Report No. 10) available that describes pertinent acceptance tests for photostimulable storage phosphor systems (computed radiography acquisition devices) [14], many of which are applicable to other digital radiography acquisition devices (charge-coupled device (CCD) and flat panel systems).

\section{Follow-up Procedures and Written Survey Reports}

The qualified medical physicist should report the findings to the physician(s), to the responsible professional(s) in charge of obtaining or providing necessary service to the equipment, and, in the case of the consulting physicist(s), to the representative of the hiring party, and, if appropriate, initiate the required service. Action must be taken immediately by verbal communication if there is imminent danger to patients or staff using the equipment due to unsafe conditions. Written survey reports should be provided in a timely manner consistent with the importance of any adverse findings.

Acknowledgments This guideline was revised according to the process described under the heading The Process for Developing ACR Practice Guidelines and Technical Standards on the ACR web site (http://www.acr.org/guidelines) by the Guidelines and Standards Committees of the Commissions on Medical Physics and General, Small, and Rural Practice in collaboration with the AAPM, and the SIIM.

Collaborative Committee-members represent their societies in the initial and final revision of this guideline

ACR

Thomas G. Ruckdeschel, MS, Co-Chair

Michael J. Flynn, PhD

Nicholas J. Hangiandreou, PhD

AAPM

Aaron K. Jones, PhD

S. Jeff Shepard, MS, FAAPM 


\section{$\underline{\text { SIIM }}$}

Katherine P. Andriole, $\mathrm{PhD}$, Co-Chair

Elizabeth Krupinski, $\mathrm{PhD}$

J. Anthony Seibert, PhD, FACR

Alisa Walz-Flannigan, $\mathrm{PhD}$

ACR Guidelines and Standards Committee-Medical Physics-ACR

Committee responsible for sponsoring the draft through the process.

Tariq A. Mian, PhD, FACR, Chair

Maxwell R. Amurao, PhD, MS

Chee-Wai Cheng, $\mathrm{PhD}$

Laurence E. Court, $\mathrm{PhD}$

Richard A. Geise, PhD, FACR

Nicholas J. Hangiandreou, PhD

Bruce E. Hasselquist, $\mathrm{PhD}$

Ralph P. Lieto, MS

Jeffrey P. Limmer, MSc

Janelle Lira Park, MD

Doug Pfeiffer, MS

Christopher J. Watchman, $\mathrm{PhD}$

Gerald A. White, Jr., MS, FACR

John W. Winston, MS

James Hevezi, PhD, FACR, Chair, Commission

ACR Guidelines and Standards Committee-GSR-ACR Commit-

tee responsible for sponsoring the draft through the process.

Matthew S. Pollack, MD, FACR, Chair

John F. AufderHeide, MD, FACR

Lawrence R. Bigongiari, MD, FACR

John E. DePersio, MD, FACR

Ronald V. Hublall, MD

Stephen M. Koller, MD

Brian S. Kuszyk, MD

Serena L. McClam, MD

James M. Rausch, MD, FACR

Geoffrey G. Smith, MD, FACR, Chair, Commission

Comments Reconciliation Committee

Mark J. Adams, MD, MBA, FACR, CSC Co-Chair

Mahadevappa Mahesh, MS, PhD, FACR, CSC Co-Chair

Katherine P. Andriole, $\mathrm{PhD}$

Kimberly E. Applegate, MD, MS, FACR

Howard B. Fleishon, MD, MMM, FACR

Michael J. Flynn, PhD

Aaron K. Jones, $\mathrm{PhD}$

Nicholas J. Hangiandreou, $\mathrm{PhD}$

James M. Hevezi, PhD, FACR

Elizabeth Krupinski, PhD

Paul A. Larson, MD, FACR

Tariq A. Mian, PhD, FACR

Debra L. Monticciolo, MD, FACR

Thomas G. Ruckdeschel, MS

J. Anthony Seibert, PhD, FACR

S. Jeff Shepard, MS

Julie K. Timins, MD, FACR

Alisa Walz-Flannigan, $\mathrm{PhD}$

\section{References}

1. Grayscale softcopy presentation state storage. Rosslyn: Digital Imaging and Communications in Medicine; 1999. Supplement 33

2. Clunie DA: Designing and implementing a PACS aware DICOM image object for digital X-ray, mammography, and intraoral applications. Proceedings of SPIE. In: Blaine GJ, Horii SC Eds. Medical Imaging 1999 PACS Design and
Evaluation: Engineering and Clinical Issues. SPIE, Bellingham, WA, 1999, pp 83-89

3. Shepard SJ, Wang J, Flynn M, et al: An exposure indicator for digital radiography: AAPM Task Group 116 (executive summary). Med Phys 36:2898-2914, 2009

4. Medical electrical equipment-exposure index of digital $x$-ray imaging systems - part 1: definitions and requirements for general radiography. Geneva, Switzerland: International Electrotechnical Commission; 2008. IEC 62494-1 ed1.0

5. Seibert JA, Shelton DK, Moore EH: Computed radiography X-ray exposure trends. Acad Radiol 3:313-318, 1996

6. Goldman LW. Speed values, AEC performance evaluation, and quality control with digital receptors. In: Goldman LW, Yester MV, ed. Specifications, performance evaluations, and quality assurance of radiographic and fluoroscopic systems in the digital era. 1st edn. Medical Physics Publishing; 2004:272-297

7. Carroll OB: Fuch's radiographic exposure and quality control, 7th edition. Charles C. Thomas, Springfield, 2003

8. Hufton AP, Doyle SM, Carty HM: Digital radiography in paediatrics: radiation dose considerations and magnitude of possible dose reduction. Br J Radiol 71:186-199, 1998

9. Ballinger PW, Frank ED: Merrill's Atlas of radiographic positions and radiologic procedures, vol. I-III, 10th edition. Mosby, St. Louis, 2003

10. Willis CE, Slovis TL: The ALARA concept in pediatric CR/DRdose reduction in pediatric radiographic exams. Pediatr Radiol 34 (Suppl 3):S162-S164, 2004

11. Hall EJ: Lessons we have learned from our children: cancer risks from diagnostic radiology. Pediatr Radiol 32:700-706, 2002

12. Strauss KJ, Poznauskis L. Practical applications of CR in pediatric imaging. App Radiol (suppl):S12-S18,2005

13. Prokop M, Neitzel U, Schaefer-Prokop C: Principles of image processing in digital chest radiography. J Thorac Imag 18:148164, 2003

14. Acceptance Testing and Quality Control of Photostimulable Storage Phosphor Imaging Systems. College Park, Md: American Association of Physicists in Medicine; 2006. AAPM Report 93; Task Group 10

\section{Appendix A}

Glossary

For additional definitions, see the ACR-AAPM-SIIM Technical Standard for Electronic Practice of Medical Imaging.

1. Analog signal — a form of information transmission in which the signal varies in a continuous manner and is not limited to discrete steps.

2. Bit (binary bit) - the smallest unit of digital information that a computing device handles. It represents off or on ( 0 or 1$)$. All data in computing devices are processed as bits or strings of bits.

3. Bit depth - the number of bits used to encode the signal intensity of each pixel of the image.

4. Charge-coupled device (CCD) - a photoelectric device that converts light information into electronic information. CCDs are commonly used in television cameras and image scanners and consist of an array of sensors 
that collect and store light as a buildup of electrical charge. The resulting electrical signal can be converted into digital values and processed in a computer to form an image.

5. Digital signal - a form of information transmission in which the signal varies in discrete steps, not in a continuous manner.

6. Exposure class - similar to the term "speed" used with SF systems. Exposure class is used to describe the nominal radiation exposure required to obtain a proper radiograph. The new term is used instead of speed to reflect the significantly different energy response that digital detectors have when compared with SF systems.

7. Exposure indicators - a quantitative method to estimate the nominal incident radiation exposure required to obtain a proper radiograph. This is a manufacturerdependent value. Fuji CR uses the sensitivity number (S number), a value that is similar to SF speed and inversely related to incident exposure. Agfa CR uses $\lg M$, the $\log$ of the median exposure determined from the segmented area on the imaging plate, which increases logarithmically with the incident exposure. Kodak CR uses exposure index, a value that represents the relative measure of the X-ray exposure in the segmented, anatomical regions of the image and increases logarithmically with the digital values in the image. Konica CR uses $S$ value, a value similar to the $S$ number with similar relationships to incident exposure, but estimated and calculated in a different manner compared with Fuji. Imaging Dynamics (a DR company) uses $f$-number, a relative value based upon the same concept as photography in describing relative light intensity ranges, where in this situation, negative values represent lower exposures and positive values represent higher exposures than the desired "nominal" exposure. Other manufacturers have their own unique exposure value estimates. Users of a particular digital system must understand the meaning of the incident exposure index in order to determine that the exposure was appropriate. The manner in which the vendor identifies the parts of the image that have received direct X-ray exposure or are collimated has an effect on the calculated exposure index and may lead to errors.

8. Exposure latitude - the range between the minimum and maximum exposures that will produce an acceptable range of densities for diagnostic purposes. Furthermore, it is considered the "margin for error in setting a proper technique." Exposure latitude is distinguished from the term "latitude" which is defined similarly as "the range of X-ray exposures that deliver
ODs in the usable range." Thus, for the same SF detector, the exposure latitude is necessarily a smaller ratio than the latitude, because the exposure latitude takes into account the full extent of X-ray exposures and corresponding densities within the range. Similarities in these two terms lead to errors in communication between technologists, medical physicists, and radiologists.

9. Fill factor - the ratio of the active charge collection area to the total physical space occupied by the detector element. Digital flat-panel detectors with better resolution (smaller del dimension) often have a lower fill factor and poorer charge collection efficiency.

10. Lookup table (LUT) - a table used to map image index numbers to output display values on a digital device.

11. Speed class - the concept of conventional SF speed (e.g., 100, 200, 400, 600, and 800) is applied to the incident exposure that a digital radiography device operates to produce an image of "acceptable" clinical image quality. The speed class for a digital radiography device depends on the device itself (how efficiently X-rays are absorbed and converted into a useful signal), the system gain adjustment (how much amplification is applied to the signals prior to digitization), and the examination requirements (how much exposure is required to achieve appropriate image quality; e.g., extremity radiographs are typically acquired with a 100 speed class setting, while chest Xrays are typically acquired with a 200 to 400 speed class setting). Operation of a system at a higher speed class requires less exposure to the patient but produces higher quantum mottle (noise) and lower SNR in the image (also see "exposure indicators").

Guidelines and standards are published annually with an effective date of 1 October in the year in which they were amended, revised, or approved by the ACR Council. For guidelines and standards published before 1999, the effective date was 1 January following the year in which the guideline or standard was amended, revised, or approved by the ACR Council.

Development Chronology for this Guideline

2007 (Resolution 42)

Amended 2009 (Resolution 11)

Revised 2012 (Resolution 37)

Reprinted with permission of the American College of Radiology, the American Association of Physicists in Medicine and the Society for Imaging Informatics in Medicine. 